\title{
Checkpoint inhibition in advanced gastroesophageal cancer: clinical trial data, molecular subtyping, predictive biomarkers, and the potential of combination therapies
}

\author{
Jeremy Chuang ${ }^{1}$, Joseph $\mathrm{Chao}^{2}$, Andrew Hendifar ${ }^{3}$, Samuel J. Klempner ${ }^{3,4}$, Jun Gong ${ }^{3}$ \\ ${ }^{1}$ Department of Internal Medicine, Harbor-UCLA Medical Center, Torrance, CA, USA; ${ }^{2}$ Department of Medical Oncology and Therapeutics \\ Research, City of Hope Comprehensive Cancer Center, Duarte, CA, USA; ${ }^{3}$ Samuel Oschin Comprehensive Cancer Institute, Cedars-Sinai Medical \\ Center, Los Angeles, CA, USA; ${ }^{4}$ The Angeles Clinic and Research Institute, Los Angeles, CA, USA \\ Contributions: (I) Conception and design: J Chuang, SJ Klempner; (II) Administrative support: None; (III) Provision of study material or patients: \\ None; (IV) Collection and assembly of data: All authors; (V) Data analysis and interpretation: All authors; (VI) Manuscript writing: All authors; (VII) \\ Final approval of manuscript: All authors. \\ Correspondence to: Samuel J. Klempner, MD. Director of Precision Medicine and GI Oncology, The Angeles Clinic and Research Institute, 11800 \\ Wilshire Boulevard, Los Angeles, CA 90025, USA. Email: sklempner@partners.org; Jun Gong, MD. Department of GI Oncology, Samuel Oschin \\ Comprehensive Cancer Institute, Cedars-Sinai Medical Center, 8700 Beverly Boulevard, Los Angeles, CA 90048, USA. Email: Jun.Gong@cshs.org.
}

\begin{abstract}
The development of checkpoint inhibitors has redefined the treatment paradigm for advanced gastroesophageal cancer. While recent developments have improved clinical outcomes, the prognosis for the disease remains meager. In this review, we discuss the rationale and detail the results from recent phase I-III trials supporting the activity of PD-1 inhibitors. Specifically, we highlight the seminal clinical trials leading to the FDA approval of pembrolizumab for advanced gastroesophageal cancer. Finally, we review the current understanding and future considerations of molecular subtyping and predictive biomarkers to help guide therapy and the promise of combination therapy to further improve the efficacy of checkpoint inhibitors.
\end{abstract}

Keywords: Gastric cancer; immunotherapy; programmed cell death 1 (PD-1); pembrolizumab; esophageal cancer; biomarker

Received: 23 February 2019; Accepted: 31 July 2019; Published: 27 August 2019.

doi: $10.21037 / \operatorname{tgh} .2019 .08 .04$

View this article at: http://dx.doi.org/10.21037/tgh.2019.08.04

\section{Introduction}

Engagement of the programmed cell death 1 (PD-1) receptor by its ligand, programmed death ligand 1 (PD-L1), has been demonstrated in studies to downregulate T-cell mediated immune responses (1-4). Early preclinical evidence supported the use of PD-1/PD-L1 inhibition as a potential mechanism for cancer immunotherapy as cancer cells have been known to evade host immune defenses with activation of PD-1/PD-L1 $(3,5,6)$. Results from early clinical trials of humanized monoclonal IgG4 antibodies targeting PD-1 and PD-L1 in patients with advanced solid tumors led to the first US Food and Drug Administration (FDA)-approved PD-1 inhibitors, pembrolizumab and nivolumab (7-9). Cancers of the stomach and esophagus are responsible for
$>60 \%$ of total cases and deaths worldwide with an estimated 1.5 million cases of gastroesophageal cancer leading to 1.1 million deaths globally in 2012 (10). Despite sequencing of available active systemic agents, prognosis remains poor in advanced disease with a median overall survival (OS) of $8-10$ months where approximately $2 / 3$ of patients with gastroesophageal cancer develop metastatic disease during the course of their disease (11). Even with the increasing prevalence of $\mathrm{PD}-1 / \mathrm{PD}-\mathrm{L} 1$ inhibitors in cancer treatment, it was not until late 2017 when the FDA first approved the use of PD-1 inhibition in advanced gastroesophageal cancer (12). In this review, we discuss the rationale and results thus far available from phase I-III trials supporting the activity of PD-1 inhibitors in advanced or metastatic 
gastroesophageal cancer. In addition, we highlight the clinical development of PD-1 inhibitors leading up to the recent approval of pembrolizumab in advanced gastroesophageal cancer. Finally, we will review the current molecular understanding and potential role of molecular subtyping to guide future therapy and the promise of combination therapy to further improve the efficacy of checkpoint inhibition.

\section{Rationale for immune checkpoint blockade in gastroesophageal cancer}

Seminal studies by The Cancer Genome Atlas (TCGA) proposed a new framework in gastroesophageal cancer with 4 subtypes in gastric cancer and 3 molecular subtypes in esophageal cancer based on comprehensive genomic profiling $(13,14)$. Notably, Epstein-Barr virus (EBV)positive subtypes $(8.8 \%)$ were characterized by PD-L1/ PD-L2 amplification and $21.7 \%$ of cases demonstrated microsatellite instability (MSI) suggesting that checkpoint inhibition may potentially be a viable approach in gastric cancer.

PD-L1 expression on tumor and immune cells (ICs) infiltrating tumor assessed by immunohistochemistry (IHC) has increasingly been shown to predict for tumor response to $\mathrm{PD}-1 / \mathrm{PD}-\mathrm{L} 1$ inhibitors [odds ratio (OR) 2.26; $95 \%$ confidence interval $(\mathrm{CI}), 1.85-2.75, \mathrm{P}<0.001]$ across all tumor types (15). A number of meta-analyses have suggested that PD-L1 expression may represent a poor prognostic marker in gastrointestinal (GI) cancers including gastric cancer (16-23). In an initial large series of GI cancer patients, the rate of PD-L1 expression $\geq 10 \%$ was nearly $50 \%$ irrespective of method of IHC evaluation used (17). Although PD-L1 overexpression was seen in as high as $74 \%$ of gastric cancers and $78 \%$ of esophageal cancers (all stages) in one series, another meta-analysis reported PD-L1 positive rates of $14.32-69.40 \%$ in gastric cancer patients (all stages) likely owing to geographic variation, discrepancies in disease characteristics and tumor stage, differences in IHC evaluation method, and variation in cut-off values for positivity or overexpression $(19,22)$. In metastatic gastric cancer patients only, $\mathrm{PD}-\mathrm{L} 1$ positivity ( $\geq 1 \%$ expression on tumor/ICs) was observed to be $8.4 \%$ on tumor cell (TC) and $65.3 \%$ on IC in a prospective cohort analyzed by the 22C3 PharmDx assay (24).

PD-L1 expression alone may not be adequate in predicting response to $\mathrm{PD}-1 / \mathrm{PD}-\mathrm{L} 1$ inhibition given a proportion of $\mathrm{PD}-\mathrm{L} 1$-expressing tumors that fail to respond to therapy (25). MSI status or DNA mismatch repairdeficiency (MMR-D) has been the only other biomarker to define an FDA-approved indication (May 23, 2017) for PD-1 blockade in advanced gastroesophageal cancer (26). In contemporary meta-analyses of gastric cancer patients (across all stages), MSI was identified in $8.5-37.8 \%$, again with heterogeneity likely attributable to disease characteristics and methodology (27-29). The rate of MSI (defined as tumors lacking either MLH1, MSH2, PMS2, or MSH6 expression) in a prospective observational cohort of metastatic gastric cancer patients has been shown to be $6.2 \%$ (24). As evidenced in these biomarker analyses, there is growing support the utility of checkpoint inhibition in gastroesophageal cancer.

\section{Phase I trials}

A number of phase I trials have assessed the role of PD-1/PD-L1 inhibitors in patients with advanced gastroesophageal cancer (7,30-42) (Table 1). KEYNOTE 012 was a multicenter open-label trial that assessed the toxicity profile and efficacy of pembrolizumab in previously treated gastric cancer with PD-L1 expression (30). Sixtyfive of 140 patients ( $40 \%$ ) screened positive for PD-L1. In this study, the authors observed $5 / 13$ patients $(13 \%)$ with grade $\geq 3$ adverse reactions. Overall response rate (ORR) of $22 \%$ was driven exclusively by partial responses with median progression-free survival (PFS) 1.9 months (95\% CI, 1.8-3.5) and median OS 11.4 months (95\% CI, 5.7not reached). The findings of this study were promising given the improved OS in comparison to single-agent chemotherapy and the lack of unexpected toxicities.

A number of different phase I trials have observed the manageable toxicity profile of a variety of experimental PD-1/PD-L1 inhibitors. In one study, the authors reported that the PD-L1 inhibitor MPDL-3280A achieved an ORR of $18 \%$ with $13 \%$ of patients experiencing grade $\geq 3$ adverse events most commonly fatigue (31). Of the 141 patients in the study, $18 \%$ of the study participants demonstrated PD$\mathrm{L} 1$ expression. The presence of PD-L1 in tumor-infiltrating immune cells may correlate with response to checkpoint inhibition $(\mathrm{P}=0.007)$. Preliminary results from the $\mathrm{PD}-1$ inhibitor BGB-A317 have also almost demonstrated promising findings (32). In this open-label phase I study of 55 patients with recurrent or refractory gastroesophageal cancer, the authors did not observe any grade $\geq 3$ adverse events related to treatment. Disease-control rate (DCR) was reported to be $32 \%$ with 3 patients reported have 
Table 1 Phase I clinical trials of PD-1 inhibitors in advanced gastroesophageal cancer

\begin{tabular}{|c|c|c|c|c|c|}
\hline$n$ & Primary tumor & Doses & Primary endpoint & Results & Ref. \\
\hline 277 & $\begin{array}{l}\text { Multiple cancer } \\
\text { types }\end{array}$ & $\begin{array}{l}\text { MPDL-3280A } 20 \text { mg/kg every } \\
3 \text { weeks }\end{array}$ & $\begin{array}{l}\text { Safety and } \\
\text { tolerability }\end{array}$ & $\begin{array}{l}13 \% \text { grade } \geq 3 \text { AEs: fatigue }(n=5) \text {; increased } \\
\text { ALT, increased AST, hypoxia ( } n=1 \text { per } \\
\text { AE); asthenia, dyspnea, myalgia, anemia, } \\
\text { hyperglycemia, hyponatremia, cardiac } \\
\text { tamponade, hypophosphatemia, tumor lysis } \\
\text { syndrome ( } n=2 \text { per AE); nausea, headache, } \\
\text { influenza-like illness, pain, vomiting ( } n=1 \text { per } \\
\text { AE); ORR } 18 \% \text { overall; } 26 \% \text { of melanoma, } \\
21 \% \text { of NSCLC, } 13 \% \text { of RCC, } 13 \% \text { other }\end{array}$ & (31) \\
\hline 151 & $\begin{array}{l}\text { Multiple cancer } \\
\text { types }\end{array}$ & $\begin{array}{l}\text { MEDI4736 } 10 \mathrm{mg} / \mathrm{kg} \text { IV every } \\
2 \text { weeks for } 12 \text { months }\end{array}$ & $\begin{array}{l}\text { Safety and } \\
\text { efficacy }\end{array}$ & $\begin{array}{l}7 \% \text { grade } 3 \geq A E s \text { with } 13 \% \text { fatigue, } 8 \% \\
\text { nausea, } 6 \% \text { rash, } 5 \% \text { vomiting, and } 5 \% \\
\text { pyrexia }\end{array}$ & (33) \\
\hline 83 & $\begin{array}{l}\text { Esophageal } \\
\text { (KEYNOTE-028) }\end{array}$ & $\begin{array}{l}\text { Pembrolizumab } 10 \mathrm{mg} / \mathrm{kg} \\
\text { every } 2 \text { weeks for up to } 2 \text { years } \\
\text { or until disease progression }\end{array}$ & Safety and ORR & ORR 30\% (95\% Cl, 13-53\%) & (34) \\
\hline 28 & GEA & $\begin{array}{l}\text { Ramucirumab } 8 \mathrm{mg} / \mathrm{kg} \text { on days } \\
1 \& 8 \text { with pembrolizumab } 200 \\
\text { mg on day } 1 \text { every } 3 \text { weeks }\end{array}$ & $\begin{array}{l}\text { Safety and } \\
\text { tolerability }\end{array}$ & $\begin{array}{l}61 \% \text { grade } \geq 3 \text { adverse events with } 14 \% \\
\text { hypotension, } 11 \% \text { diarrhea, and } 7 \% \text { liver } \\
\text { function abnormalities; DCR } 68 \% \text {; median } \\
\text { PFS } 5.3 \text { months ( } 95 \% \mathrm{Cl}, 3.2-11)\end{array}$ & (35) \\
\hline 43 & $\begin{array}{l}\text { GEA, } \\
\text { hepatocellular }\end{array}$ & $\begin{array}{l}\text { SHR-1210 } 200 \text { mg every } \\
2 \text { weeks and apatinib } \\
125-500 \text { mg once daily until } \\
\text { unacceptable toxicity or } \\
\text { disease progression }\end{array}$ & $\begin{array}{l}\text { Safety and } \\
\text { efficacy }\end{array}$ & $\begin{array}{l}60.6 \% \text { grade } \geq 3 \mathrm{AE} ; 15.2 \% \text { hypertension, } \\
15.2 \% \text { increased aspartate aminotransferase; } \\
\text { ORR } 30.8 \% \text { overall ( } 95 \% \mathrm{Cl}, 17.0-47.6 \%) ; \\
5 / 25 \text { gastroesophageal patients }(20 \%) \text { with } \\
\text { PR, median PFS } 2.9 \text { months } \\
(95 \% \mathrm{Cl}, 2.5-4.2 \text { months) }\end{array}$ & (38) \\
\hline 57 & GEA (JAVELIN) & $\begin{array}{l}\text { Avelumab } 10 \text { mg/kg every } \\
2 \text { weeks }\end{array}$ & $\begin{array}{l}\text { Safety and } \\
\text { efficacy }\end{array}$ & $\begin{array}{l}3 / 40 \text { patients }(7.5 \%) \text { grade } \geq 3 \mathrm{AE} \text { including } \\
\text { alanine aminotransferase increase, anemia, } \\
\text { and hyponatremia; ORR } 10.0 \%(95 \% \mathrm{Cl} \text {, } \\
2.8-23.7 \%) \text {; median OS } 9.1 \text { months } \\
\text { (95\% Cl, 7.2-11.2 months) }\end{array}$ & (39) \\
\hline
\end{tabular}

Table 1 (continued) 
Table 1 (continued)

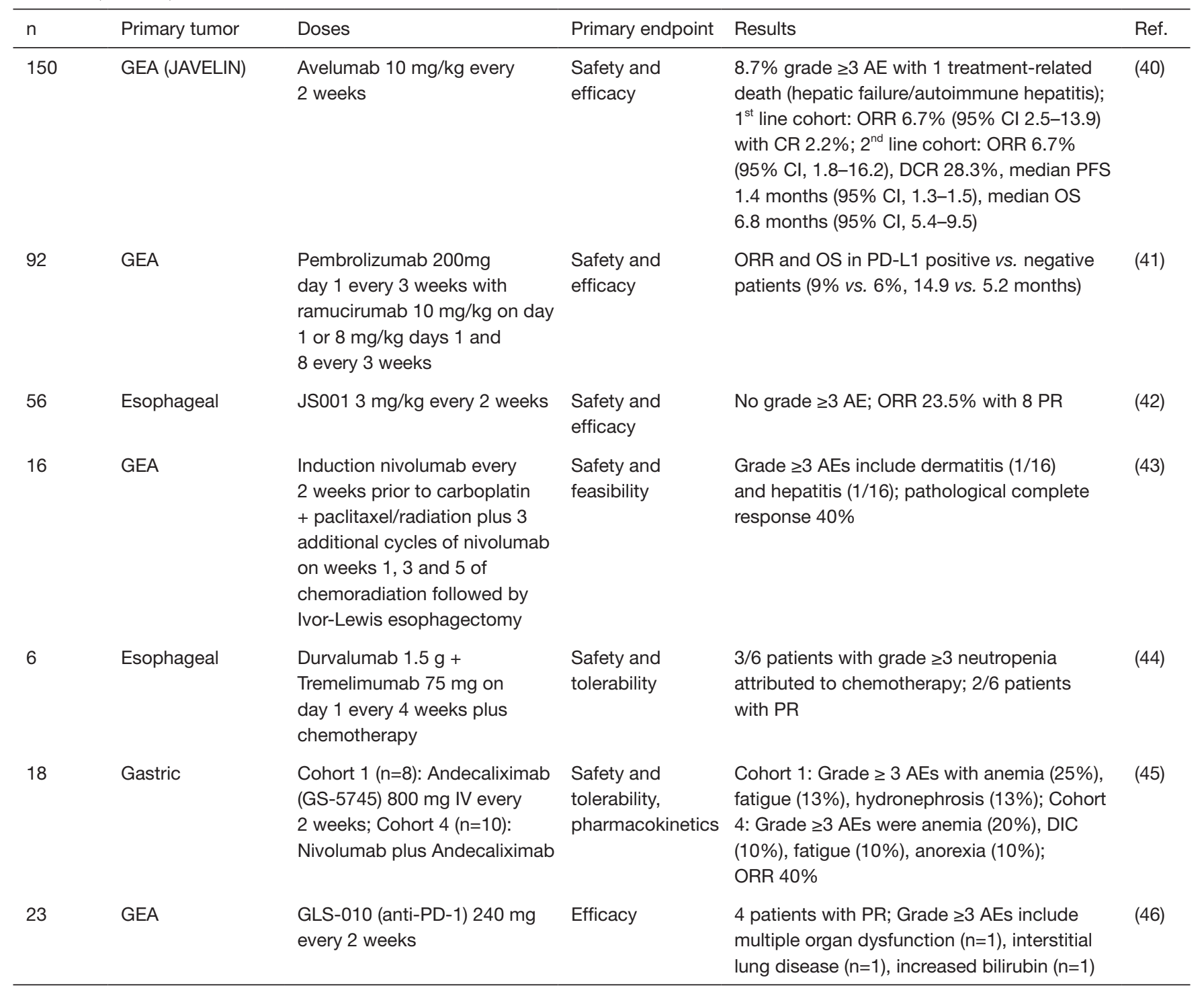

GEA, gastroesophageal adenocarcinoma; PD, progressive disease; ORR, overall response rate; Cl, confidence interval; AE, adverse event; $\mathrm{DCR}$, disease-control rate; $\mathrm{CR}$, complete response; $\mathrm{PR}$, partial response.

achieved partial response. The PD-1 inhibitor MEDI4736 (durvalumab) has also demonstrated a tolerable side effect profile in the treatment of multiple cancer types including NSCLC, gastroesophageal, hepatocellular carcinoma, melanoma (cutaneous and ocular), pancreatic, SCCHN and triple negative breast cancer with $7 \% \geq$ grade 3 AEs most commonly from fatigue (33). The study is currently ongoing however authors report observing tumor shrinkage across a variety of malignancies. Another study sought to evaluate the safety and efficacy of the anti-PD-1 inhibitor JS001 in 56 Chinese patients with advanced esophageal cancer (42). PD-L1 positively was $21.4 \%$. ORR was $23.5 \%$ with 8 partial responses. No grade $3 \geq$ AEs were observed. The authors did not observe any difference in clinical response in patients with or without PD-L1 expression.

KEYNOTE 028 evaluated the safety and efficacy of pembrolizumab in 83 patients with esophageal malignancy (34). Thirty-seven of the cohort (45\%) demonstrated PD-L1-positive tumors. ORR was $30 \%$ (95\% CI, $13-53 \%$ ) with PFS of $30 \%$ and $22 \%$ at 6 and 12 months, respectively. Median duration of response was 40 weeks. 
Recently, there has also been a strong interest in combination therapy with $\mathrm{PD}-1 / \mathrm{PD}-\mathrm{L} 1$ inhibition. In a phase I study, the authors evaluated the safety and tolerability of ramucirumab (anti-VEGFR-2) plus pembrolizumab in patients with gastroesophageal malignancy (35). Twenty-eight patients were enrolled in this study; $68 \%$ were PD-L1 positive. Seventeen patients $(61 \%)$ experienced grade $\geq 3$ adverse events most commonly hypertension (14\%). Disease control rate was $68 \%$ while median PFS was 5.3 months. Another phase I study evaluated the safety and tolerability of ramucirumab plus durvalumab in 29 patients with gastroesophageal malignancy (36). Forty-eight percent had PD-L1 $\geq 25 \%$ expression. Thirty-five percent of patients experienced grade $3 \geq$ treatment-related AEs with most common AEs including hypertension, fatigue, headache, diarrhea, pyrexia, and decreased appetite. Five patients (17\%) achieved confirmed partial response; median PFS was 2.6 months. In another study, the authors evaluated the safety and tolerability of ramucirumab with pembrolizumab in 92 patients with advanced gastroesophageal cancer (41). ORR and OS in PD-L1 positive versus PD-L1 negative patients were $9 \%$ and 14.9 months compared to $6 \%$ and 5.2 months, respectively. From the results of the study, it appears that combination therapy may provide greater benefit to patients with PD-L1 positive cancers compared with PDL1 negative patients.

A phase I study from China evaluated the evaluated the safety and efficacy of SHR-1210 in 30 patients with advanced gastroesophageal cancer. Of the 28 Chinese patients who had PD-L1 status evaluated, 13 patients (46.4\%) had PD-L1-positive tumors. Two patients with grade $3 \geq$ treatment-related AEs with one patient with grade 5 interstitial lung disease. ORR was $7 / 30$ patients $(23.3 \%)$ (95\% CI, 9.9-42.3\%) with one patient with complete response and 6 patients with partial responses. ORR in PD-L1-positive tumors [3 of 13 (23.1\%)] which was similar to patients with PD-L1-negative tumors [4 of 15 (26.7\%); $\mathrm{P}=1.000$ ]. Median was PFS 8.0 weeks (95\% CI, 7.9-8.1 weeks). Combination strategies utilizing SHR-1210 are also being studied. In one study, the authors assessed the safety and SHR-1210 (anti-PD-1 antibody) and apatinib (VEGFR2 inhibitor) in 43 patients with gastroesophageal and hepatocellular cancer (38). Twenty out of thirty-three patients $(60.6 \%)$ who achieved recommended phase II dose (RP2D) experienced a grade $\geq 3$ treatment-related adverse event most commonly hypertension (15\%) or liver function abnormalities (15\%). ORR observed was 30.8\% (95\% CI,
17.0-47.6\%); 5/25 gastroesophageal patients achieved partial responses.

Avelumab is a PD-L1 inhibitor of clinical interest that has been studied in a number of trials. In the Japanese JAVELIN phase I trial, the authors evaluated the safety and efficacy of avelumab in 151 Japanese patients with advanced gastroesophageal cancer (39). PD-L1 expression was not assessed in this study. Three out of forty patients experienced $(7.5 \%)$ grade $3 \mathrm{AE}$ including anemia, alanine aminotransferase increase, and hyponatremia. The ORR was $10.0 \%$ (95\% CI, $2.8-23.7 \%$ ) while median OS was 9.1 months (95\% CI, 7.2-11.2 months). In another JAVELIN study, the authors evaluated the role of avelumab in 150 patients with advanced gastroesophageal cancer without or with disease progression after first line therapy (1L and 2L cohort, respectively) (40). Eight point seven percent of all patients experienced grade $\geq 3 \mathrm{AE}$ with 1 treatment-related death secondary to hepatic failure/ autoimmune hepatitis. In the $1 \mathrm{~L}$ cohort, ORR was $6.7 \%$ (95\% CI, 2.5-13.9) with a complete response of $2.2 \%$. In the $2 \mathrm{~L}$ cohort, ORR was $6.7 \%$ (95\% CI, 1.8-16.2), DCR $28.3 \%$, median PFS 1.4 months (95\% CI, 1.3-1.5), and median OS 6.8 months (95\% CI, 5.4-9.5).

\section{Phase II trials}

Several phase II clinical trials have studied the efficacy of PD-1/PD-L1 blockade in advanced gastroesophageal cancers (Table 2). CheckMate 032 was a phase I/II study that studied the clinical impact of nivolumab, an anti-PD-1 monoclonal antibody, along with ipilimumab, a CTLA4 inhibitor in 160 patients with advanced gastroesophageal malignancy (47). Patients were randomized to one of three cohorts: nivolumab alone, nivolumab $1 \mathrm{mg} / \mathrm{kg}$ with ipilimumab $3 \mathrm{mg} / \mathrm{kg}(\mathrm{N} 1+\mathrm{I} 3)$, or nivolumab $3 \mathrm{mg} / \mathrm{kg}$ with ipilimumab $1 \mathrm{mg} / \mathrm{kg}(\mathrm{N} 3+\mathrm{I} 1)$. Thirty-nine out of 127 evaluable patients $(31 \%)$ demonstrated PD-L1 positivity $(\geq 1 \%)$. In general, the ORRs were greater in cancers with PD-L1 expression (nivolumab alone 19\%, N1+I3 40\%, N3+I1 $23 \%$ ) however there remain a modest response in $\mathrm{PD}-$ L1 deficient malignancies as well (12\% nivolumab alone, $22 \% \mathrm{~N} 1+\mathrm{I} 3,0 \% \mathrm{~N} 3+\mathrm{I} 1)$. In addition, PD-L1 expression appears to be correlated with improved 12 -month OS in the $\mathrm{N} 1+\mathrm{I} 3$ cohort (50\%) compared to nivolumab alone (34\%) and N3+I1 (23\%) cohorts. However, the small sample size of PD-L1 positive cancers in each cohort makes it difficult draw definitive conclusions about whether PD-L1 status alone may predict who will benefit from checkpoint 


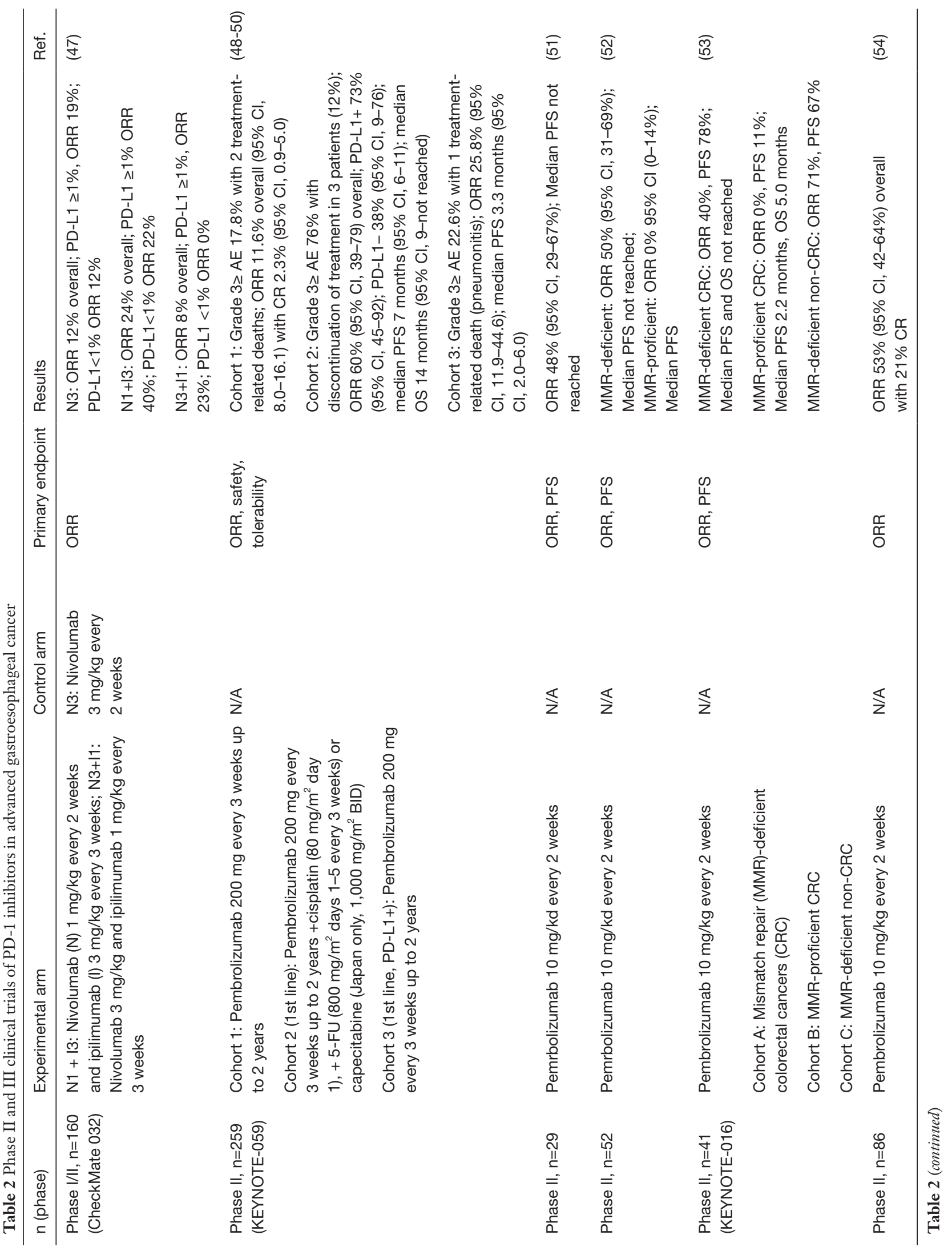




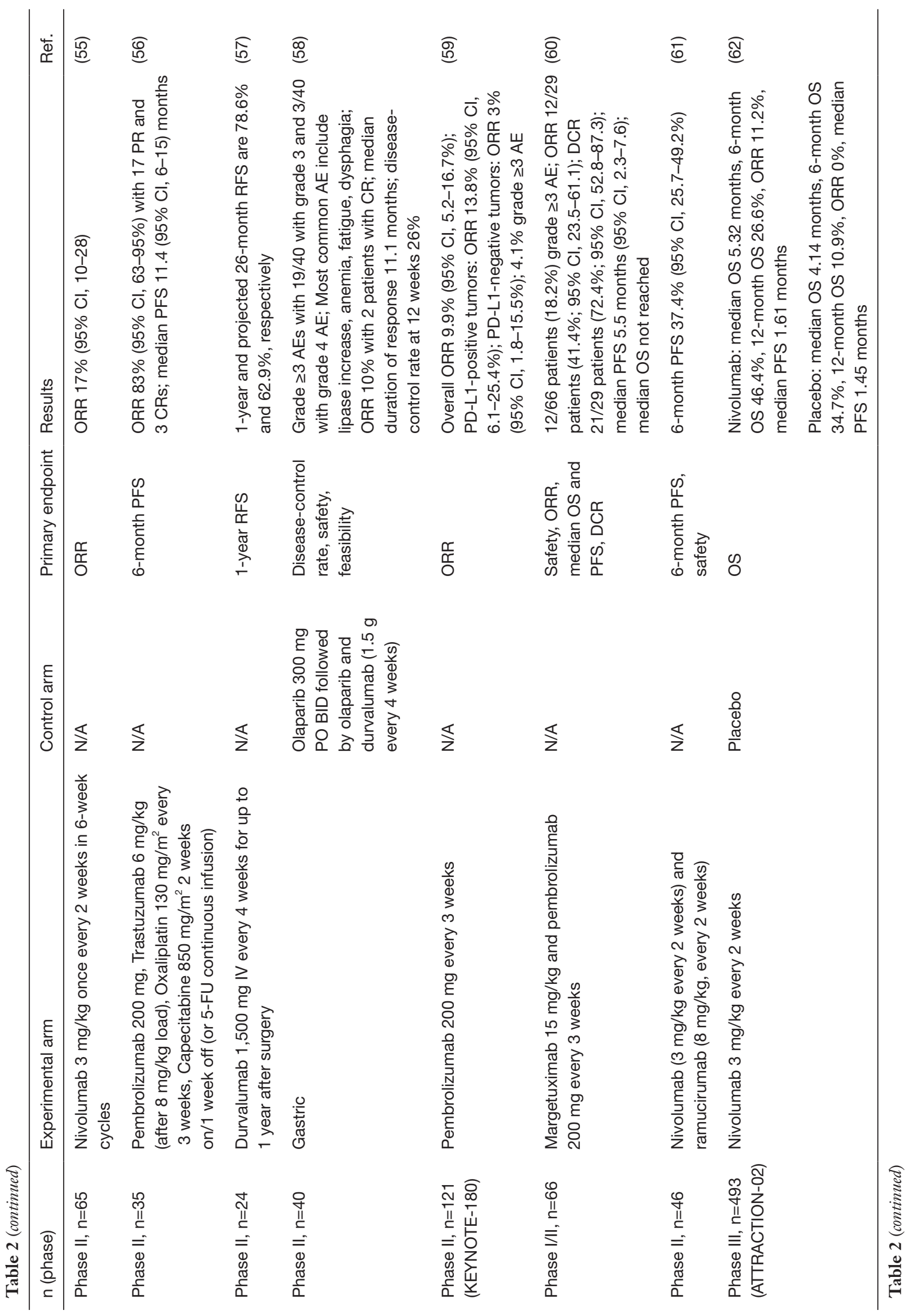




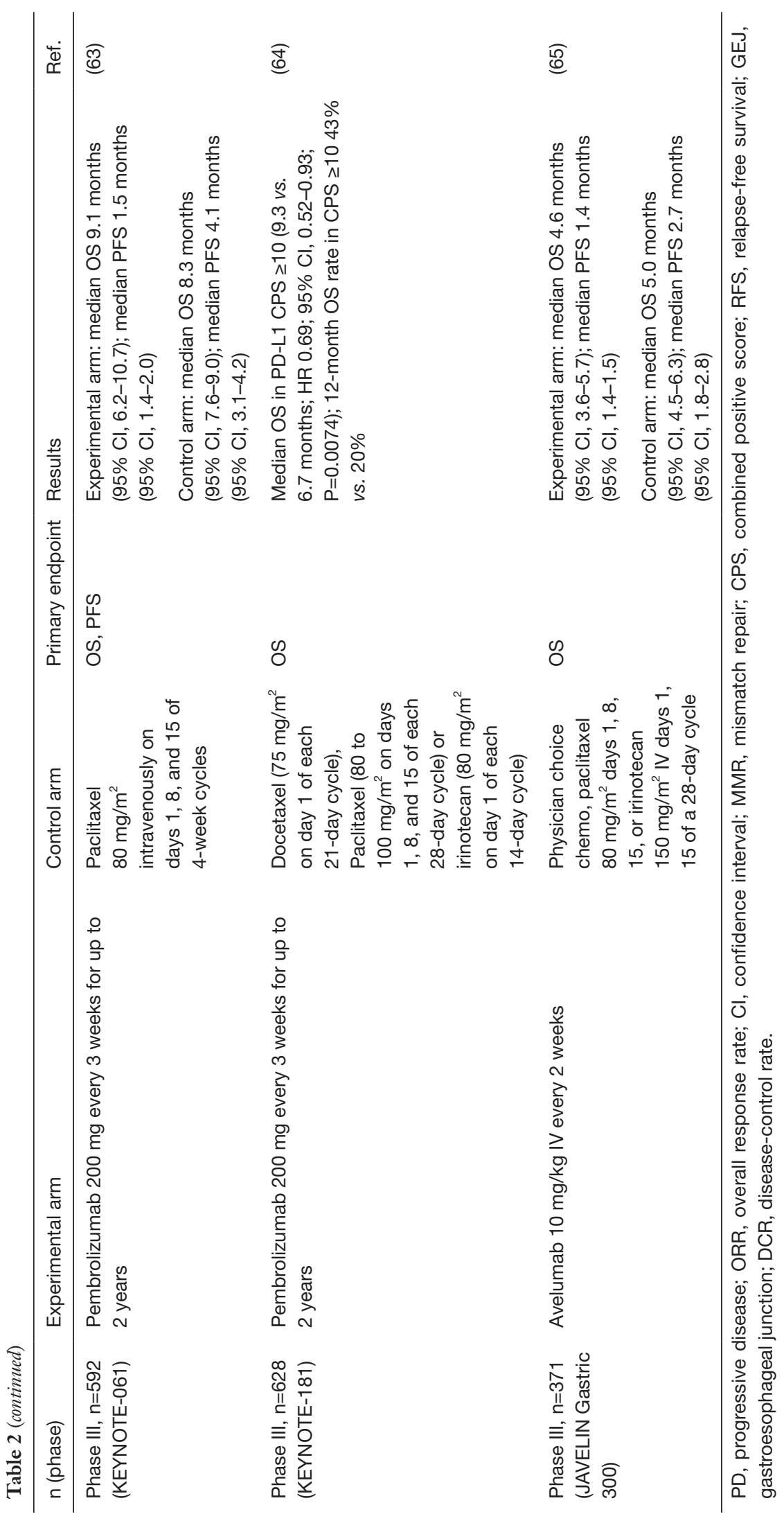


inhibition.

KEYNOTE 059 was a seminal study that paved the way for FDA approval of pembrolizumab in refractory gastroesophageal cancer with $\mathrm{PD}-\mathrm{L} 1$ expression on September 22, 2017 (48-50). This open-label, multicenter clinical study of 259 patients with gastroesophageal malignancy and evidence of disease progression despite prior therapy. Patients were enrolled into 3 cohorts: pembrolizumab for patients who previously received two lines of therapy (cohort 1), pembrolizumab with cisplatin and either 5-fluorouracil or capecitabine (cohort 2), and pembrolizumab for patients with PD-L1 positivity $(\geq 1 \%)$ (cohort 3). Within cohort 1 , the authors reported that patients with PD-L1 expression $(148 / 259,57.1 \%)$ had an ORR $15.5 \%$ (95\% CI, 10.1-22.4\%) in comparison to 6.4\% (95\% CI, 2.6-12.8\%) in PD-L1 negative tumors (109/259, $42.1 \%$ ), which suggests that PD-L1 negative status did not preclude observing a response to therapy. Of the 259 patients enrolled, 7 patients or $4 \%$ of the cohort were found to be MSI-high. Four out of seven patients (57.1\%) demonstrated an objective response (95\% CI, 18.4-90.1\%). In contrast, patients with non-MSI-high malignancies had an ORR of $9.0 \%$. While MSI-high tumors demonstrated a robust response, the sample size was small and most of the responses within the study were amongst non-MSI high patients.

Preliminary results from cohort 2 and 3 are also available $(48,49)$. Cohort 2 reported on 25 patients who received first-line chemotherapy in addition to pemrbolizumab. The authors observed an ORR of $60 \%$ overall with an ORR in PD-L1+ of $73 \%$ (95\% CI, 45-92) while achieving an ORR of PD-L1- 38\% (95\% CI, 9-76) PD-L1- tumors. These results suggest that $\mathrm{PD}-\mathrm{L} 1$ status may identify individuals who may benefit from PD-L1 inhibition, however the sample size in the study population is too small to draw any definitive conclusions. Cohort 3 enrolled 31 patients to receive pembrolizumab as first-line therapy with PD-L1 positivity. The authors observed an ORR of $25.8 \%$ (95\% CI, 11.9-44.6) with complete response in $3.2 \%$ of patients. Median PFS 3.3 months (95\% CI, 2.0-6.0). Seven patients (22.6\%) experienced grade $\geq 3$ adverse events; one patient had a treatment-related death from pneumonitis. Overall, these preliminary results demonstrate promising activity and a manageable toxicity profile of pembrolizumab in patients with PD-L1 positive gastroesophageal tumors.

There have also been studies to study the clinical impact of PD-1 inhibition in the setting of mismatch repair deficiency. It has been hypothesized that the burden of immunogenic antigens from mismatch repair deficits may favor the use of checkpoint inhibition to mobilize the immune system against cancer cells. In a phase II study of 29 patients with cancers of multiple types including gastric malignancy, the authors observed an ORR of ORR 48\% (95\% CI, 29-67\%) (51). Median PFS was not reached. The results of these studies ultimately led to the tissue-agnostic approval of pembrolizumab in treatment-refractory malignancies. Another phase II trial assessed the impact of pembrolizumab in 52 patients with or without mismatch repair deficiency (MMR) in colon cancers (52). In tumors with MMR-deficient, ORR was $50 \%$ (95\% CI, 31-69\%) while tumors with MMRproficient had an ORR 0\% (95\% CI, 0-14\%). In a phase II study (KEYNOTE-016) assessing the clinical impact of pembrolizumab in patients with colorectal cancer with or without MMR deficiency, the authors observed an ORR of $40 \%$ and $0 \%$, respectively. In addition, the authors included a third cohort of non-colorectal MMRdeficient tumors, which demonstrated an ORR of $71 \%$. The study was further expanded to include 86 patients with multiple cancer types with MMR-deficiency (54) and found to have an ORR of $53 \%$ (95\% CI, 42-64\%) along with a CR rate of $21 \%$. Within this study, 5 patients had gastroesophageal cancer and had a CR rate of $60 \%$. The promising results from this study along with results from KEYNOTE-012, -164, -028, and -158 contributed to the accelerated approval of pembrolizumab on May 23, 2017 for treatment of microsatellite instability-high (MSI-H) malignancies. Other studies including other PD-1 inhibitors are listed in the Table 2 (55).

A recent phase II clinical study with 35 patients with previously untreated metastatic HER2-positive gastroesophageal tumors with pembrolizumab and trastuzumab along with chemotherapy with the primary endpoint of evaluating 6-month PFS (56). Fourteen patients enrolled had cancer in the esophagus, 12 in the gastroesophageal junction, and 9 with gastric. PD-1 expression status was positive in 14 patients; no tumors demonstrated MSI. ORR was $83 \%$ (95\% CI, 63-95\%) with 17 partial responses and 3 complete responses. Median PFS was 11.4 months (95\% CI, 6-15). In addition, patients in the study tolerated the regimen; common grade $\geq 3$ adverse effects include decreased lymphocyte count (12\%). Overall, this study suggests potential synergy for this combination regimen in gastroesophageal tumors and represents a significant improvement compared to the response rate of frontline trastuzumab plus 5-FU/platinum chemotherapy. 
In subgroup analysis, it did not appear that PD-L1 status conferred an advantage in OS or PFS. An ongoing phase III trial (KEYNOTE-811; NCT03615326) utilizing this combination for frontline therapy will help to clarify the impact on OS.

The Big Ten investigators recently presented the results demonstrating the safety and efficacy of durvalumab in patients with locally advanced gastroesophageal cancer in a phase II trial (57). The trial enrolled 24 patients with gastroesophageal cancer; 14 patients had gastroesophageal junction adenocarcinoma while 10 had distal esophageal adenocarcinoma. The 1-year and projected relapse-free survival were $78.6 \%$ and $62.9 \%$, respectively. In addition, the treatment was well-tolerated with $5 / 18$ patients (20.8\%) experiencing grade $\geq 3$ adverse events, most commonly diarrhea, hepatitis, hypoglycemia, and encephalopathy. A recent phase II study of 40 patients with relapsed gastric cancer was administered the PARP inhibitor olaparib followed by combination olaparib and durvalumab to evaluate the DCR, safety, and tolerability (58). The authors reported the regimen was tolerated with $22 / 40$ patients (55\%) experiencing grade $\geq 3$ adverse reactions most commonly anemia, fatigue, dysphagia, and hyponatremia. The ORR was $10 \%$ with 2 complete and partial responses observed. Median duration of response to therapy was 11.1 months with 12 -week DCR $26 \%$. Because the responses occurred during combination therapy, there may potentially be synergistic effects between the two therapies.

KEYNOTE 180 was a phase II study of 121 patients with advanced, metastatic esophageal cancer to evaluate the efficacy and toxicity profile pembrolizumab (59). Of the 121 patients, 48 (47.9\%) had tumors positive for PDL1. Overall ORR was 9.9\% (95\% CI, 5.2-16.7\%), 13.8\% (95\% CI, 6.1-25.4\%) amongst PD-L1-positive tumors, and $6.3 \%$ (95\% CI, 1.8-15.5\%) from PD-L1-negative tumors (4 of 63 ). The regimen was well tolerated with $12.4 \%$ experiencing a grade $\geq 3$ adverse events. Only one treatment-related death from pneumonitis was observed. Other studies that were recently presented at ASCO GI are listed in Table $1(60,61)$.

\section{Phase III trials}

Several phase III trials have studied the clinical impact of PD-1/PD-L1 inhibitors in advanced gastroesophageal cancers (Table 2). In a randomized, double-blind, placebocontrolled phase III trial (ATTRACTION-02), 493 patients from Japan, South Korea, and Taiwan with refractory gastroesophageal caner were randomized $(2: 1)$ to receive either nivolumab $3 \mathrm{mg} / \mathrm{kg}$ or placebo with the primary endpoint of evaluating OS. Twenty-six patients (14\%) were found to be PD-L1 positive (62). In comparison to the placebo cohort, the experimental arm had improved median OS of 5.26 months (95\% CI, 4.60-6.37) compared to 4.14 months (95\% CI, 3.42-4.86). In addition, 12-month OS rates were $26.2 \%$ (95\% CI, 20.7-32.0) in the experimental arm compared with $10.9 \%$ (95\% CI, 6.2$17.0)$ in the placebo cohort. There were 5 patients with treatment-related deaths. In an exploratory analysis, PDL1 tumor status did not appear to significantly impact OS. The median OS in tumors with PD-L1 positivity in experimental $v s$. placebo arm was 5.22 months (95\% CI, 2.79-9.36) vs. 3.83 (95\% CI, 0.79-9.36) in comparison to PD-L1 negative tumors with median OS of 6.05 months (95\% CI, 4.83-8.54) vs. 4.19 months (95\% CI, 3.02-6.93). Given the small sample size, it is difficult to conclude how PD-L1 status may impact the choice of therapy. The results of this study led to the approval of nivolumab in Japan for use as third-line therapy in advanced gastroesophageal cancer.

KEYNOTE-061 was a multicenter phase III trial enrolled 592 patients with refractory, PD-L1 positive gastroesophageal cancer to receive either pembrolizumab $200 \mathrm{mg}$ every 3 weeks for up to 2 years or standard-dose paclitaxel (63). Three hundred ninety-five out of 592 patients $(66.7 \%)$ were found to be PD-L1 positive. Median OS was 9.1 months (95\% CI, 6.2-10.7) while Median PFS 1.5 months (95\% CI, 1.4-2.0) in experimental arm while median OS and PFS were 8.3 months (95\% CI, 7.6-4.1 months) (95\% CI, 3.1-4.2), respectively in control arm. Forty-two out of 294 patients (14\%) experienced grade $\geq 3$ adverse events in experimental arm compared to $96 / 276$ patients (35\%) in control arm. Response rate were similar with $16 \%$ (95\% CI, 11-22\%) in experimental arm compared to $14 \%$ (95\% CI, 9-19\%) in control arm. While pembrolizumab appeared to have a more manageable toxicity profile than paclitaxel, it did not appear to significantly improve OS. In subgroup analyses, the authors did observe improved response rates in patients with increased PD-L1 combined positive scores (CPS) $\geq 10(24.5 \%$ vs. $9.1 \%)$ and MSI-H tumors irrespective of PD-L1 CPS score (46.7\% vs. 16.7\%). As such, there may be potential role for MSI status and PD-L1 expression in identifying patients who may benefit from checkpoint inhibition.

KEYNOTE-181 was a randomized, phase III study that 
demonstrated significantly improved OS in pembrolizumab as second-line therapy compared to chemotherapy in a study of 628 patients with advanced esophageal cancers with PD-L1 expression (64). Both patients with squamous cell or adenocarcinoma histologies were recruited including Siewert I adenocarcinomas of the GE junction. Of the 628 patients enrolled, 222 patients had PD-L1 CPS $\geq 10$. In patients with CPS $\geq 10$, the median OS was 9.3 vs. 6.7 months (HR 0.69; 95\% CI, 0.52-0.93; $\mathrm{P}=0.0074$ ) and 12 -month OS rate was $43 \%$ vs. $20 \%$. Statistical significance was not reached for OS in the study amongst patients with squamous cell carcinoma (SCC), however there was still an improvement in OS for patients with SCC (HR 0.78; 95\% CI, 0.63-0.96). In addition, pembrolizumab was welltolerated. These findings support the use of pembrolizumab as potentially a new standard of care for second-line therapy in esophageal cancers.

\section{Discussion}

Immune checkpoint inhibitors have made great strides in becoming implemented as standard of care options in refractory gastroesophageal cancers. Currently PD-1 inhibitors are approved for use as third-line therapy for PD-L1 positive gastroesophageal cancer (12) and in treatment-refractory metastatic gastroesophageal cancers that are MSI-H (26). As the number of trials investigating checkpoint blockade in gastroesophageal cancer grows $($ Tables 1,2$)$, it is important to validate predictive biomarkers that will identify patients who will benefit from immunotherapy in gastroesophageal cancer treatment. MSI-H and PD-L1 are probably the earliest and most described predictive biomarkers. A recent prospective study observed that PD-L1 expression was commonly observed in patients with KRAS mutation $(\mathrm{P}=0.002)$, PIK3CA mutation $(\mathrm{P}=0.020)$, MMR-deficiency $(\mathrm{P}<0.001)$ while PD-L1 expression on immune cells were associated with EBV ( $\mathrm{P}=0.034)$ (24). These findings support screening gastroesophageal tumors for MMR-deficiency and EBV status along with PD-L1 expression.

However, MSI-H and PD-L1+ gastroesophageal tumors still comprise only a fraction of the overall population of gastroesophageal cancer patients in need of further investigation to identify patients who will benefit from immunotherapy. Recent efforts are beginning to explore other predictive biomarkers to predict response to checkpoint blockade in gastroesophageal cancer (66-72). Given the varied outcomes from patients with similar staging in gastric cancer, the 5-factor immunoscore was developed in a retrospective study of 879 patients using IHC to develop a prediction model for enhance prognosis and survival (66). The score includes the assessment of 5 immune features: $\mathrm{CD} 3$ invasive margin (IM), CD3 center of tumor (CT), CD8IM, CD45ROCT, and CD66bIM. Higher scores correlated with better 5-year OS and diseasefree survival In addition, the combination of CT and IM along with TNM appeared to provide better prognostic information than TNM staging alone with higher scores correlated with better 5 -year OS and disease-free survival. This study also demonstrated that patients with higher immunoscores may benefit from adjuvant therapy in patients with stage II/III gastric cancer. Additional studies have identified that the combination of PD-L1 expression and immunoscore can provide prognostic information (67). Specifically, patients with PD-L1 positive expression demonstrated better prognosis with higher immunoscores than with lower scores. In addition, the combined PD-L1 status and immunoscore was an independent prognostic marker for OS in patients with MSI-H gastric cancer. Other prediction models have been developed including the four-factor immunoscores devised based on hazard ratios on the basis of PD-L1+ immune cells (IC), PD-L1+ TC, PD-1hi, and CD8More (68). This scoring system allows for patients to be stratified to low, medium, and high-risk groups with different survival outcomes.

Other biomarkers under investigation include tumorinfiltrating lymphocytes (TILs). In one study of 398 patients, the authors observed that increased TIL density was correlated with reduced risk of disease progression and may be associated with a survival benefit (69). In addition, the study also found that PD-L1 expression and EBV+ was associated with increased TIL density. In addition, MSI has also been correlated with PD-L1 expression and high TILs (71). In addition to immunoscores, other prediction models are currently being studied including the hostimmune response index (HIERI). A recent validation study performed gene sequencing to identify 29 immune genes of interest within gastric cancer to stratify patients into 3 groups with prognostic significance (70). The first cluster which was defined by elevated PD-L1 expression, EBV positivity, MSI, and increased TIL density demonstrated the best prognosis while the cluster defined by EBV negativity, microsatellite stability, and low TIL density had poorer outcomes. Of note, half of the immune-active cluster was EBV negative and microsatellite stable suggesting a 
segment of this population may potentially candidates to checkpoint inhibition. T-cell inflamed gene expression profile (GEP) is another molecular biomarker that may predict response to PD-L1 therapy (72). In a study of 574 gastric tumors, an 18-gene signature was measured from extracted tumor RNA; high GEP was associated with tumor grade and clinical stage $(\mathrm{P}<0.0001)$. In addition, GEP was associated with improved OS (HR 0.46; 95\% CI, 0.32-0.66) and weakly correlated with PD-L1 expression [Spearman $\mathrm{r}=0.24(\mathrm{P}<0.0001)]$. Further investigation will be necessary to validate GEP as a potential biomarker to predict response to immunotherapy.

A recent study provides important insights to the potential role for circulating tumor DNA (ctDNA) and other molecular markers in predicting response to treatment with pembrolizumab (73). In this prospective phase II trial, 61 patients with metastatic gastric cancer who received pembrolizumab demonstrated favorable responses particularly in patients with EBV and MSI-H tumors with ORR of $85.7 \%$ and $100 \%$, respectively. In addition, PD-L1 expression was associated with improved responses to therapy with ORR of $50 \%$ compared to $0 \%$ in PD-L1 negative tumors $(\mathrm{P}<0.001)$. Furthermore, ctDNA levels correlated well to radiographic response and in posthoc analysis may also predict PFS and risk for disease progression. With increasing use of checkpoint inhibitors in gastroesophageal cancer and in other malignancies, in general, the American Society of Clinical Oncology (ASCO) and Society for Immunotherapy of Cancer (SITC) recently presented recommendations for IO trial reporting standard on efficacy and toxicity (74). The report highlighted 12 key recommendations: (I) reporting the criteria to evaluate for response to therapy; (II) including spider or swimmer plots to evaluate response to therapy; (III) defining disease control rates; (IV) criteria to treat patients beyond disease progression; (V) reporting number of patients treated beyond disease progression and significant clinical events including new toxicity and efficacy; (VI) Kaplan-Meier analyses for PFS and OS; (VII) reporting the clinical diagnoses of the toxicity and its symptomatology (VIII) defining the different types of adverse events (i.e., immunerelated adverse events) and why they were selected for reporting; (IX) reporting toxicity by grade; (X) reporting interventions to manage toxicity; (XI) reporting acuity and duration of toxicity; and (XII) discussing the rationale for selection of the dose and sequence for treatment regimens. Lastly, while we continue our search for markers to select optimal candidates for checkpoint inhibitor in advanced gastroesophageal cancer, development of novel strategies combining checkpoint blockade with other treatment modalities are being explored to enhance on the efficacy of single-agent checkpoint inhibitors in this setting. Chemotherapy and checkpoint blockade combinations represent an intuitive combination strategy, for example, in gastroesophageal cancer given that cytotoxic therapies have been a foundation for metastatic GEJ treatment for decades.

Chemotherapy has potentially multiple immunologic effects ranging from eliminating immunosuppressive cells such as regulatory $\mathrm{T}$ cells or macrophages to enhancing the ability of $T$ cells to detect tumor antigens. Recent findings from Wanderley et al. observed that paclitaxel may act as a TLR4 agonist to modulate and reprogram tumor-associated macrophages towards a M-1 phenotype and thereby potentially improve the efficacy of checkpoint inhibition (75). While these findings lay the groundwork for combination therapy, further studies will help to elucidate the mechanisms that underlie the immunomodulation of chemotherapeutic agents to optimize their potent effects with checkpoint inhibition. A number of studies have sought to evaluate the efficacy of checkpoint inhibitors in combination with chemotherapeutic agents or radiotherapy (43,76-78). One phase II trial found the PDL1 inhibitor LY3300054 (LY) alone or in combination with ramucirumab or merestinib in 38 patients with advanced, refractory solid tumors demonstrated antitumor activity with one partial response in each cohort (76). Another phase II trial evaluated SHR-1220 with radiotherapy in 20 patients with newly diagnosed locally advanced esophageal SCC intolerable or refused chemoradiotherapy and observed 2 patients (11.1\%) with complete responses and 13 patients $(72.2 \%)$ with partial responses (77). Other studies demonstrating encouraging results include the combination of DKN-01, a monoclonal antibody against DKK1, a potential NK-cell mediator and pembrolizumab (78). Early reporting from 13 patients with advanced gastroesophageal adenocarcinomas, the authors observed that there was 1 partial response amongst 9 evaluable patients. In addition, patients who traditionally were associated with poor response to PD-L1 inhibition (KRAS amplification, PDL1 negativity, and MSS) demonstrated a potential clinical benefit when utilizing this combination of therapy.

\section{Conclusions}

The overwhelming evidence clearly supports a role for immunotherapy in gastric and esophageal cancers 
though many unanswered questions remain. The optimal placement of immunotherapy (neoadjuvant, perioperative, maintenance, front line metastatic, all lines, etc.) in the continuum of care continues to evolve and we await important findings from several ongoing phase III trials. Early data has also suggested that immunotherapy activity may be improved via combinations with chemotherapy, other immune targeting agents, trastuzumab, and agents acting on angiogenic pathways. Harmonization among trial reporting and better baseline and longitudinal molecular assessments will aid in refining the optimal patient selection and may one day identify subsets who may only need single agent versus those who may require combination therapies. We have attempted to provide a comprehensive report on available prospective datasets and eagerly await future data.

\section{Acknowledgments}

SJ Klempner is supported by the Howard H. Hall fund for esophagogastric cancer research.

\section{Footnote}

Conflicts of Interest: J Chao has received research support (institutional), consulting, and speaker fees from Merck and consulting fees from Lilly. SJ Klempner has received speaker fees from Merck and consulting fees from Lilly, Boston Biomedical, Astellas, and Foundation Medicine, Inc. SJ Klempner holds stock/equity in TP Therapeutics. The other authors have no conflicts of interest to declare.

Ethical Statement: The authors are accountable for all aspects of the work in ensuring that questions related to the accuracy or integrity of any part of the work are appropriately investigated and resolved.

\section{References}

1. Dong H, Zhu G, Tamada K, et al. B7-H1, a third member of the B7 family, co-stimulates T-cell proliferation and interleukin-10 secretion. Nat Med 1999;5:1365-9.

2. Freeman GJ, Long AJ, Iwai $Y$, et al. Engagement of the PD-1 immunoinhibitory receptor by a novel B7 family member leads to negative regulation of lymphocyte activation. J Exp Med 2000;192:1027-34.

3. Greenwald RJ, Freeman GJ, Sharpe AH. The B7 family revisited. Annu Rev Immunol 2005;23:515-48.

4. Nishimura H, Nose M, Hiai H, et al. Development of lupus-like autoimmune diseases by disruption of the PD-1 gene encoding an ITIM motif-carrying immunoreceptor. Immunity 1999;11:141-51.

5. Dong H, Strome SE, Salomao DR, et al. Tumor-associated B7-H1 promotes T-cell apoptosis: a potential mechanism of immune evasion. Nat Med 2002;8:793-800.

6. Iwai $Y$, Ishida $M$, Tanaka $Y$, et al. Involvement of PD-L1 on tumor cells in the escape from host immune system and tumor immunotherapy by PD-L1 blockade. Proc Natl Acad Sci U S A 2002;99:12293-7.

7. Brahmer JR, Tykodi SS, Chow LQ, et al. Safety and activity of anti-PD-L1 antibody in patients with advanced cancer. N Engl J Med 2012;366:2455-65.

8. Patnaik A, Kang SP, Rasco D, et al. Phase I Study of Pembrolizumab (MK-3475; Anti-PD-1 Monoclonal Antibody) in Patients with Advanced Solid Tumors. Clin Cancer Res 2015;21:4286-93.

9. Topalian SL, Hodi FS, Brahmer JR, et al. Safety, activity, and immune correlates of anti-PD-1 antibody in cancer. $\mathrm{N}$ Engl J Med 2012;366:2443-54.

10. Torre LA, Siegel RL, Ward EM, et al. Global Cancer Incidence and Mortality Rates and Trends--An Update. Cancer Epidemiol Biomarkers Prev 2016;25:16-27.

11. Shah MA. Update on metastatic gastric and esophageal cancers. J Clin Oncol 2015;33:1760-9.

12. Fashoyin-Aje L, Donoghue M, Chen H, et al. FDA Approval Summary: Pembrolizumab for Recurrent Locally Advanced or Metastatic Gastric or Gastroesophageal Junction Adenocarcinoma Expressing PD-L1. Oncologist 2019;24:103-9.

13. Cancer Genome Atlas Research Network. Comprehensive molecular characterization of gastric adenocarcinoma. Nature 2014;513:202-9.

14. Cancer Genome Atlas Research Network. Integrated genomic characterization of oesophageal carcinoma. Nature 2017;541:169-75.

15. Khunger M, Hernandez AV, Pasupuleti V, et al. Programmed cell death 1 (PD-1) ligand (PD-L1) expression in solid tumors as a predictive biomarker of benefit from PD-1/PD-L1 axis inhibitors: A systematic review and meta-analysis. JCO Precision Oncology 2017. doi: 10.1200/PO.16.00030.

16. Zhang M, Dong Y, Liu H, et al. The clinicopathological and prognostic significance of PD-L1 expression in gastric cancer: a meta-analysis of 10 studies with 1,901 patients. Sci Rep 2016;6:37933.

17. Huang B, Chen L, Bao C, et al. The expression status and prognostic significance of programmed cell death 1 ligand 
1 in gastrointestinal tract cancer: a systematic review and meta-analysis. Onco Targets Ther 2015;8:2617-25.

18. Dai C, Wang M, Lu J, et al. Prognostic and predictive values of PD-L1 expression in patients with digestive system cancer: a meta-analysis. Onco Targets Ther 2017;10:3625-34.

19. Gu L, Chen M, Guo D, et al. PD-L1 and gastric cancer prognosis: A systematic review and meta-analysis. PLoS One 2017;12:e0182692.

20. Xu F, Feng G, Zhao H, et al. Clinicopathologic Significance and Prognostic Value of B7 Homolog 1 in Gastric Cancer: A Systematic Review and Meta-Analysis. Medicine (Baltimore) 2015;94:e1911.

21. Liu YX, Wang XS, Wang YF, et al. Prognostic significance of PD-L1 expression in patients with gastric cancer in East Asia: a meta-analysis. Onco Targets Ther 2016;9:2649-54.

22. Wu P, Wu D, Li L, et al. PD-L1 and Survival in Solid Tumors: A Meta-Analysis. PLoS One 2015;10:e0131403.

23. Zhang $W$, Pang $Q$, Yan C, et al. Induction of PD-L1 expression by epidermal growth factor receptor-mediated signaling in esophageal squamous cell carcinoma. Onco Targets Ther 2017;10:763-71.

24. Kawazoe A, Shitara K, Kuboki Y, et al. Clinicopathological features of 22C3 PD-L1 expression with mismatch repair, Epstein-Barr virus status, and cancer genome alterations in metastatic gastric cancer. Gastric Cancer 2019;22:69-76.

25. Gibney GT, Weiner LM, Atkins MB. Predictive biomarkers for checkpoint inhibitor-based immunotherapy. Lancet Oncol 2016;17:e542-51.

26. Boyiadzis MM, Kirkwood JM, Marshall JL, et al. Significance and implications of FDA approval of pembrolizumab for biomarker-defined disease. J Immunother Cancer 2018;6:35.

27. Polom K, Marano L, Marrelli D, et al. Meta-analysis of microsatellite instability in relation to clinicopathological characteristics and overall survival in gastric cancer. Br J Surg 2018;105:159-67.

28. Choi YY, Bae JM, An JY, et al. Is microsatellite instability a prognostic marker in gastric cancer? A systematic review with meta-analysis. J Surg Oncol 2014;110:129-35.

29. Zhu L, Li Z, Wang Y, et al. Microsatellite instability and survival in gastric cancer: A systematic review and metaanalysis. Mol Clin Oncol 2015;3:699-705.

30. Muro K, Chung HC, Shankaran V, et al. Pembrolizumab for patients with PD-L1-positive advanced gastric cancer (KEYNOTE-012): a multicentre, open-label, phase 1b trial. Lancet Oncol 2016;17:717-26.

31. Herbst RS, Soria JC, Kowanetz M, et al. Predictive correlates of response to the anti-PD-L1 antibody MPDL3280A in cancer patients. Nature 2014;515:563-7.

32. Desai J, Millward M, Chao Y, et al. Preliminary results from subsets of patients (pts) with advanced gastric cancer (GC) and esophageal carcinoma (EC) in a dose-escalation/ expansion study of BGB-A317, an anti-PD-1 monoclonal antibody (mAb). Ann Oncol 2017;28:v122-41.

33. Segal NH, Antonia SJ, Brahmer JR, et al. Preliminary data from a multi-arm expansion study of MEDI4736, an antiPD-L1 antibody. J Clin Oncol 2014;32:3002.

34. Doi T, Piha-Paul SA, Jalal SI, et al. Safety and Antitumor Activity of the Anti-Programmed Death-1 Antibody Pembrolizumab in Patients With Advanced Esophageal Carcinoma. J Clin Oncol 2018;36:61-7.

35. Chau I, Penel N, Arkenau HT, et al. Safety and antitumor activity of ramucirumab plus pembrolizumab in treatment naïve advanced gastric or gastroesophageal junction (G/ GEJ) adenocarcinoma: Preliminary results from a multidisease phase I study (JVDF). J Clin Oncol 2018;36:101.

36. Bang YJ, Golan T, Lin CC, et al. Interim safety and clinical activity in patients (pts) with locally advanced and unresectable or metastatic gastric or gastroesophageal junction (G/GEJ) adenocarcinoma from a multicohort phase I study of ramucirumab (R) plus durvalumab (D). J Clin Oncol 2018;36:92.

37. Huang J, Mo H, Zhang W, et al. Promising efficacy of SHR-1210, a novel anti-programmed cell death 1 antibody, in patients with advanced gastric and gastroesophageal junction cancer in China. Cancer 2019;125:742-9.

38. Xu JM, Zhang Y, Jia R, et al. Anti-PD-1 Antibody SHR-1210 combined with Apatinib for Advanced Hepatocellular Carcinoma, Gastric or Esophagogastric Junction Cancer: An Open-label, Dose Escalation and Expansion Study. Clin Cancer Res 2018. doi: 10.1158/1078-0432.CCR-18-2484

39. Doi T, Iwasa S, Muro K, et al. Phase 1 trial of avelumab (anti-PD-L1) in Japanese patients with advanced solid tumors, including dose expansion in patients with gastric or gastroesophageal junction cancer: the JAVELIN Solid Tumor JPN trial. Gastric Cancer 2019;22:817-27.

40. Chung HC, Arkenau HT, Lee J, et al. Abstract CT111: Avelumab (anti-PD-L1) as first-line maintenance (1L mn) or second-line (2L) therapy in patients with advanced gastric or gastroesophageal junction cancer (GC/GEJC): updated phase Ib results from the JAVELIN Solid Tumor trial. Cancer Res 2018;78:CT111-CT.

41. Herbst RS, Chau I, Petrylak DP, et al. Activity of ramucirumab $(\mathrm{R})$ with pembrolizumab $(\mathrm{P})$ by $\mathrm{PD}-\mathrm{L} 1$ 
expression in advanced solid tumors: Phase $1 \mathrm{a} / \mathrm{b}$ study in later lines of therapy. J Clin Oncol 2018;36:3059.

42. Xu RH, Wang F, Shi J, et al. Recombinant humanized antiPD-1 monoclonal antibody (JS001) as salvage treatment for advanced esophageal squamous cell carcinoma: Preliminary results of an open-label, multi-cohort, phase Ib/II clinical study. J Clin Oncol 2018;36:116.

43. Catenacci DV, Park H, Uronis HE, et al. Margetuximab (M) plus pembrolizumab (P) in ERBB2-amplified PD-L1+ gastroesophageal adenocarcinoma (GEA) post trastuzumab (T). J Clin Oncol 2018;36:4030.

44. Lee DH, Keam H, Kato B, et al. Evaluation of safety and tolerability of durvalumab (D) and tremelimumab (T) in combination with first-line chemotherapy in patients (pts) with esophageal squamous-cell carcinoma (ESCC). J Clin Oncol 2019;37:146.

45. Yamaguchi K, Satoh T, Muro K, et al. Phase 1 b study of andecaliximab (GS-5745, ADX) as monotherapy and in combination with nivolumab (nivo) in Japanese subjects with gastric or GEJ adenocarcinoma. J Clin Oncol 2019;37:137

46. Lin S, Gong J, Xu Y, et al. Gls-010, a novel anti-PD-1 $\mathrm{mAb}$ in Chinese advanced gastrointestinal tumor: Result of a phase Ib clinical trial. J Clin Oncol 2019;37:125.

47. Janjigian YY, Bendell J, Calvo E, et al. CheckMate-032 Study: Efficacy and Safety of Nivolumab and Nivolumab Plus Ipilimumab in Patients With Metastatic Esophagogastric Cancer. J Clin Oncol 2018;36:2836-44.

48. Wainberg ZA, Jalal S, Muro K, et al. KEYNOTE-059 Update: Efficacy and safety of pembrolizumab alone or in combination with chemotherapy in patients with advanced gastric or gastroesophageal (G/GEJ) cancer. Ann Oncol 2017;28:Abstr nr LBA28_PR.

49. Catenacci Daniel V, Wainberg Z, Fuchs Charles S, et al. LBA-009KEYNOTE-059 cohort 3: safety and efficacy of pembrolizumab monotherapy for first-line treatment of patients (pts) with PD-L1-positive advanced gastric/ gastroesophageal (G/GEJ) cancer. Ann Oncol 2017;28. doi: 10.1093/annonc/mdx302.008.

50. Fuchs CS, Doi T, Jang RW, et al. Safety and Efficacy of Pembrolizumab Monotherapy in Patients With Previously Treated Advanced Gastric and Gastroesophageal Junction Cancer: Phase 2 Clinical KEYNOTE-059 Trial. JAMA Oncol 2018;4:e180013.

51. Diaz LA, Uram JN, Wang H, et al. Programmed death-1 blockade in mismatch repair deficient cancer independent of tumor histology. J Clin Oncol 2016;34:Abstr nr 3003.

52. Le DT, Uram JN, Wang H, et al. PD-1 blockade in mismatch repair deficient non-colorectal gastrointestinal cancers. J Clin Oncol 2016;34:Abstr nr 195.

53. Le DT, Uram JN, Wang H, et al. PD-1 Blockade in Tumors with Mismatch-Repair Deficiency. N Engl J Med 2015;372:2509-20.

54. Le DT, Durham JN, Smith KN, et al. Mismatch repair deficiency predicts response of solid tumors to PD-1 blockade. Science 2017;357:409-13.

55. Kudo T, Hamamoto Y, Kato K, et al. Nivolumab treatment for oesophageal squamous-cell carcinoma: an open-label, multicentre, phase 2 trial. Lancet Oncol 2017;18:631-9.

56. Janjigian YY, Chou JF, Simmons M, et al. First-line pembrolizumab $(\mathrm{P})$, trastuzumab $(\mathrm{T})$, capecitabine (C) and oxaliplatin (O) in HER2-positive metastatic esophagogastric adenocarcinoma (mEGA). J Clin Oncol 2019;37:62.

57. Mamdani H, Schneider BJ, Abushahin LI, et al. Safety and efficacy of durvalumab following trimodality therapy for locally advanced esophageal and GEJ adenocarcinoma: Early efficacy results from Big Ten Cancer Research Consortium study. J Clin Oncol 2019;37:5.

58. Bang YJ, Kaufman B, Geva R, et al. An open-label, phase II basket study of olaparib and durvalumab (MEDIOLA): Results in patients with relapsed gastric cancer. J Clin Oncol 2019;37:140.

59. Shah MA, Kojima T, Hochhauser D, et al. Efficacy and Safety of Pembrolizumab for Heavily Pretreated Patients With Advanced, Metastatic Adenocarcinoma or Squamous Cell Carcinoma of the Esophagus: The Phase 2 KEYNOTE-180 Study. JAMA Oncol 2019;5:546-50.

60. Catenacci DV, Lim KH, Uronis HE, et al. Antitumor activity of margetuximab (M) plus pembrolizumab (P) in patients (pts) with advanced HER2+ (IHC3+) gastric carcinoma (GC). J Clin Oncol 2019;37:65.

61. Hara H, Shoji H, Takahari D, et al. Phase I/II study of ramucirumab plus nivolumab in patients in second line treatment for advanced gastric adenocarcinoma (NivoRam study). J Clin Oncol 2019;37:129.

62. Kang YK, Boku N, Satoh T, et al. Nivolumab in patients with advanced gastric or gastro-oesophageal junction cancer refractory to, or intolerant of, at least two previous chemotherapy regimens (ONO-4538-12, ATTRACTION-2): a randomised, double-blind, placebocontrolled, phase 3 trial. Lancet 2017;390:2461-71.

63. Shitara K, Ozguroglu M, Bang YJ, et al. Pembrolizumab versus paclitaxel for previously treated, advanced gastric or gastro-oesophageal junction cancer (KEYNOTE-061): a randomised, open-label, controlled, phase 3 trial. Lancet 
2018;392:123-33.

64. Kojima T, Muro K, Francois E, et al. Pembrolizumab versus chemotherapy as second-line therapy for advanced esophageal cancer: Phase III KEYNOTE-181 study. J Clin Oncol 2019;37:2.

65. Bang YJ, Ruiz EY, Van Cutsem E, et al. Phase III, randomised trial of avelumab versus physician's choice of chemotherapy as third-line treatment of patients with advanced gastric or gastro-oesophageal junction cancer: primary analysis of JAVELIN Gastric 300. Ann Oncol 2018;29:2052-60.

66. Jiang Y, Zhang Q, Hu Y, et al. ImmunoScore Signature: A Prognostic and Predictive Tool in Gastric Cancer. Ann Surg 2018;267:504-13.

67. Kim KJ, Yang HK, Kim WH, et al. Combined prognostic effect of PD-L1 expression and immunoscore in microsatellite-unstable advanced gastric cancers. Oncotarget 2017;8:58887-902.

68. Wen T, Wang Z, Li Y, et al. A Four-Factor Immunoscore System That Predicts Clinical Outcome for Stage II/III Gastric Cancer. Cancer Immunol Res 2017;5:524-34.

69. Dai C, Geng R, Wang C, et al. Concordance of immune checkpoints within tumor immune contexture and their prognostic significance in gastric cancer. Mol Oncol 2016;10:1551-8.

70. Park C, Cho J, Lee J, et al. Host immune response index in gastric cancer identified by comprehensive analyses of tumor immunity. Oncoimmunology 2017;6:e1356150.

71. De Rosa S, Sahnane N, Tibiletti MG, et al. EBV(+) and MSI Gastric Cancers Harbor High PD-L1/PD-1 Expression and High CD8(+) Intratumoral Lymphocytes. Cancers (Basel) 2018;10. doi: 10.3390/cancers10040102.

72. Rha SY, Ku GY, Kim HS, et al. PD-L1 expression and

doi: 10.21037/tgh.2019.08.04

Cite this article as: Chuang J, Chao J, Hendifar A, Klempner SJ, Gong J. Checkpoint inhibition in advanced gastroesophageal cancer: clinical trial data, molecular subtyping, predictive biomarkers, and the potential of combination therapies. Transl Gastroenterol Hepatol 2019;4:63.
T-cell inflamed gene expression profile (GEP) in Korean and US patients (pts) with advanced gastric cancer (GC). Ann Oncol 2018;29:viii205-70.

73. Kim ST, Cristescu R, Bass AJ, et al. Comprehensive molecular characterization of clinical responses to PD-1 inhibition in metastatic gastric cancer. Nat Med 2018;24:1449-58.

74. Tsimberidou AM, Levit LA, Schilsky RL, et al. Trial Reporting in Immuno-Oncology (TRIO): an American society of clinical oncology-society for immunotherapy of cancer statement. J Immunother Cancer 2018;6:108.

75. Wanderley CW, Colon DF, Luiz JPM, et al. Paclitaxel Reduces Tumor Growth by Reprogramming TumorAssociated Macrophages to an M1 Profile in a TLR4Dependent Manner. Cancer Res 2018;78:5891-900.

76. Patnaik A, Bang YJ, Chung HC, et al. Abstract CT018: Interim safety and clinical activity in patients (pts) with advanced refractory solid tumors from a phase $\mathrm{Ia} / \mathrm{b}$ study investigating the novel anti-PD-L1 antibody (LY3300054) administered alone or in combination with other agents. Cancer Res 2018;78:CT018-CT.

77. Pang Q, Li X, Zhang W, et al. Safety and Effect of Radiation Therapy Combined with Anti-PD-1 Antibody SHR-1210 as First-Line Treatment on Patients with Intolerable Concurrent Chemoradiotherapy Esophageal Cancer: A Phase 1B Clinical Trial. Int J Radiat Oncol Biol Phys 2018;102:e39.

78. Klempner SJ, Bendell J, Meucci Villaflor V, et al. Safety and efficacy of a DKK1 inhibitor (DKN-01) in combination with pembrolizumab $(\mathrm{P})$ in patients $(\mathrm{Pts})$ with advanced gastroesophageal (GE) malignancies. Ann Oncol 2018;29:viii205-70. 